\title{
Immune Cell Function Assay
}

National Cancer Institute

\section{Source}

National Cancer Institute. Immune Cell Function Assay. NCI Thesaurus. Code C73490.

A set of laboratory assays that examine the responses of lymphoid or myeloid cells after exposure to an experimental material. 